\title{
Research on the Application of Furnace Temperature Monitoring in Hot Pressing Furnace Based on Kalman Filter Algorithm of Distribution Graphs
}

\author{
Xin Haiyan, Wang Mimi, Liu Wenbin
}

School of Electron \& Computer, Chengxian College, Southeast University, Nanjing, Jiangsu Province, 210088, P. R. China

Keywords: kalman filter; furnace temperature; distribution graphs

\begin{abstract}
Data fusion technology can effectively improve computing efficiency and reduce redundant data. In this paper, the furnace temperature of hot-pressing furnace is taken as the research object. Aiming at the small fluctuation of the fusion result of the average value and the traditional Kalman filter, an improved Kalman filter algorithm is proposed by introducing the distribution graph method, which is used to monitor the furnace temperature data. Simulation results show that the improved algorithm has good fusion effect and can effectively improve the accuracy of furnace temperature data.
\end{abstract}

\section{Structure of furnace temperature monitoring system for hot pressing furnace}

The temperature monitoring system of hot-pressing furnace is mainly composed of multi-channel temperature sensor, amplifier circuit, A/D conversion circuit, interface circuit and industrial computer, as shown in Figure 1. The industrial control computer in the system chooses OMRON industrial control computer PLC. Five temperature sensors are set at each typical temperature zone of the hot-press furnace. The temperature acquisition circuit converts the temperature signal collected by the temperature sensor into a DC voltage signal of $0-5 \mathrm{~V}$, and converts it into a digital signal by A/D conversion circuit, and sends it to the computer to eliminate the negligence. The results of consistency are obtained and data fusion is carried out. The industrial control computer can also decide the temperature control mode according to the preset and measured temperature values in each temperature zone, and control the electrified state of the heating element of the hot-press furnace through the temperature control output interface, thus realizing the closed-loop control of the temperature in each temperature zone.

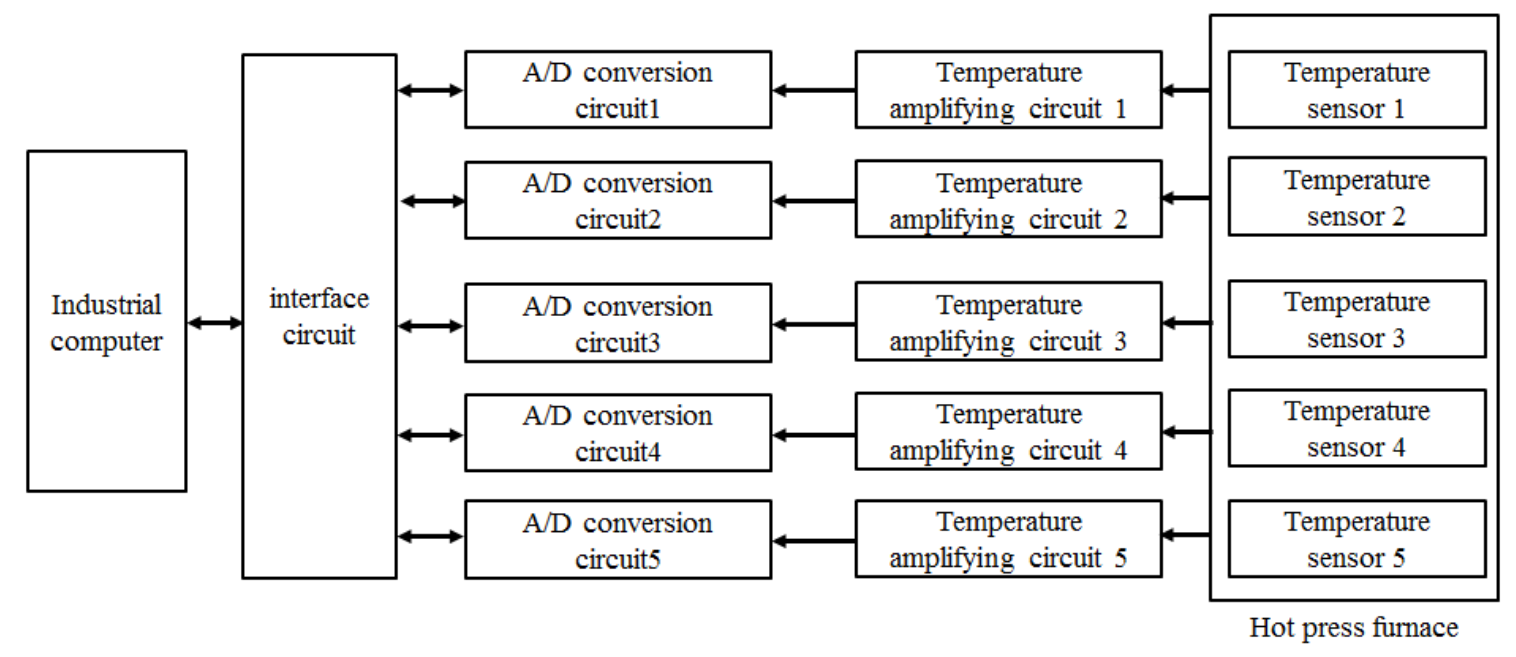

Fig. 1 Structure of furnace temperature monitoring system for hot pressing furnace

\section{The traditional Kalman filter algorithm}

Kalman filter algorithm uses recursive method to solve the linear filtering problem. It is mainly 
used for the fusion of redundant information in dynamic environment. According to the estimated value of the previous state and the observed value of the current state, the filtering method of the current state estimation is deduced ${ }^{[1,2]}$. Considering a discrete process control system, the time updates (1) and (2) of Kalman filter algorithm are shown, including the time recursive state variables and the forward recursive error covariance.

$$
\begin{aligned}
& \hat{X}_{k}=A \hat{X}_{k-1} \\
& P_{k-1}=A P_{k-1} A^{T}+Q \\
& \hat{X}_{k}=\hat{X}_{\bar{k}}+K_{k}\left(Y_{k}-\hat{X}_{\bar{k}}\right) \\
& P_{k}=\left(I-K_{k} C\right) P_{\bar{k}} \\
& K_{k}=P_{\bar{k}} C^{T}\left(C P_{\bar{k}} C^{T}+R\right)^{-1}
\end{aligned}
$$

\section{Kalman filter algorithm based on distribution graphs method}

The algorithm in this paper is as follows:

1) The measured $\mathrm{N}$ furnace temperature signals are arranged from small to large, which is the lower limit and the upper limit.

2) The median is defined as formula (6) and formula (7), the upper part is defined as the median of the interval, and the lower part is defined as the median of the interval.

$$
\begin{array}{lr}
T_{M}=T_{\frac{N+1}{2}} \quad N=2 k+1 \\
T_{M}=\frac{T_{\frac{N}{2}+1}+T_{\frac{N}{2}}}{2} & \quad N=2 k
\end{array}
$$

3) Quantile dispersion is defined as equation (8). If the distance between the measured furnace temperature and the median is greater than that of the missing data, the discriminant interval of the missing data is defined as equation (9) and (10).

$$
\begin{gathered}
d F=F_{U}-F_{L} \\
\rho_{1}=F_{L}-\frac{\beta}{2} d F \\
\rho_{2}=F_{U}+\frac{\beta}{2} d F
\end{gathered}
$$

In the formula, the constant is determined by the accuracy of the temperature measurement of the hot pressing furnace, and the general value is 1 or 2 .

Distribution graph method eliminates $50 \%$ of the deviated value interference by calculating the judgment interval, and the selection of median and quartile dispersion is independent of the size of the extreme value point, only depends on the location of the data distribution. The acquisition of effective interval has little relationship with the missing data, and the data obtained by distribution graph method is not restricted by the data distribution. Therefore, the distribution graph method has the advantages of small amount of computation, strong robustness and good real-time performance. In order to keep the dimension of the data unchanged, the estimated value will be used to replace the missing data, which can reduce the dimension judgment and improve the efficiency of 
calculation.

In this paper, the traditional Kalman filter algorithm is improved, mainly by introducing the distribution chart method. In practical application, firstly, the furnace temperature data fused by Kalman filter algorithm is collected, and the missing data is determined by the distribution chart method; the parameters of Kalman filter are re-initialized, and then Kalman filter algorithm is used to iterate repeatedly to re-carry out the temperature data fusion.

\section{Experimental results and analysis}

The data of a thermostat in a hot-pressing furnace for processing metal matrix composites of Tai'an Azure Metal Ceramic Material Co., Ltd. are selected as shown in Table (1).

Table 1 furnace temperature data sheet

\begin{tabular}{|c|c|c|c|c|c|}
\hline time(s) & sensor1 & sensor 2 & sensor 3 & sensor 4 & sensor 5 \\
\hline 10 & 24.6 & 24.7 & 25.1 & 25.3 & 24.5 \\
\hline 20 & 24.6 & 24.6 & 25.1 & 25.3 & 24.5 \\
\hline 30 & 24.5 & 24.7 & 25.1 & 25.2 & 24.4 \\
\hline 40 & 24.5 & 24.7 & 25.1 & 25.3 & 24.6 \\
\hline 50 & 24.3 & 24.7 & 25.1 & 25.3 & 24.4 \\
\hline 60 & 24.6 & 24.7 & 25.2 & 25.2 & 24.4 \\
\hline 70 & 24.5 & 24.6 & 24.9 & 25.2 & 24.5 \\
\hline 80 & 24.4 & 24.6 & 25.1 & 25.3 & 24.5 \\
\hline 90 & 24.5 & 24.5 & 24.9 & 25.3 & 24.6 \\
\hline 100 & 24.6 & 24.6 & 24.9 & 25.2 & 24.5 \\
\hline
\end{tabular}

The temperature data of the hot-pressing furnace in Table (1) are shown in Figure 2. It is obvious that the temperature data collected by each sensor do not fluctuate very much, which basically reflects the characteristics of the constant temperature furnace. However, comparing the data collected by the five sensors, the data are quite different. In order to accurately reflect the current temperature information of the constant temperature furnace, the original furnace temperature data are fused with mean value, Kalman filter and improved Kalman filter. The fusion results are shown in Figure 3.

From the comparison of the results in Figure 3, we can see that the convergence trend of the three methods is almost the same. The fusion values of the average value and Kalman filter algorithm at 40s, 50s, 60s and 70s have slight mutation. Therefore, compared with the average algorithm and Kalman filter algorithm, the fusion curve of the improved Kalman filter algorithm is smoother and accords with the temperature variation law of the constant temperature furnace.

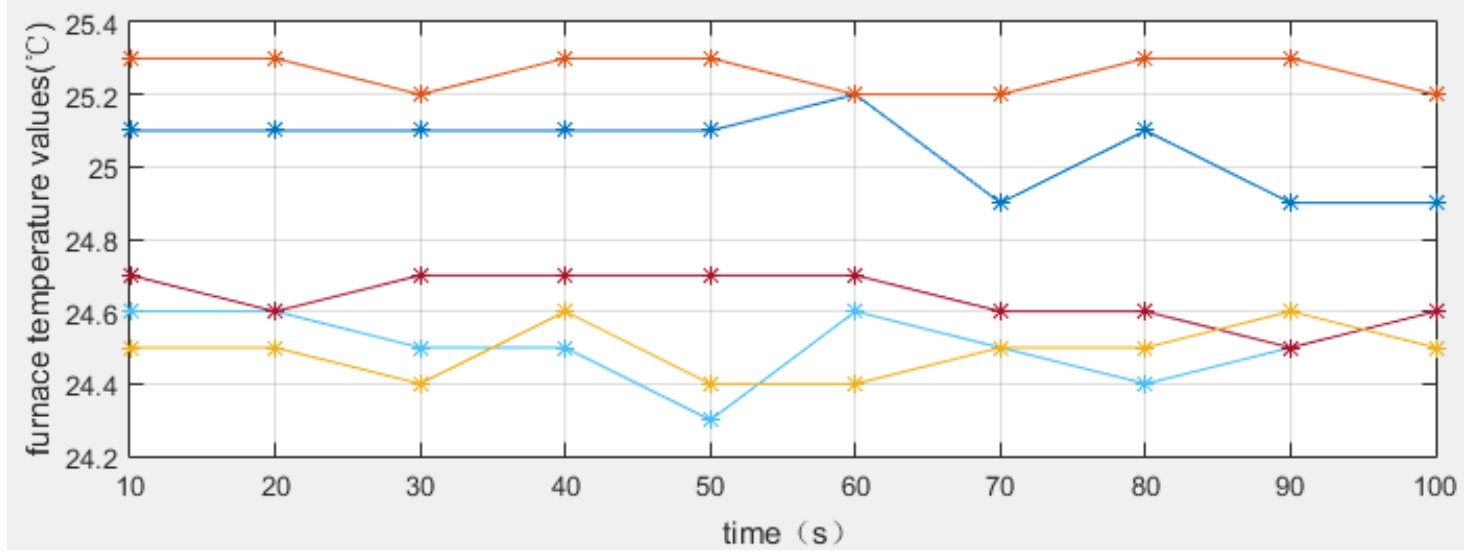

Fig. 2 Raw data of furnace temperature 


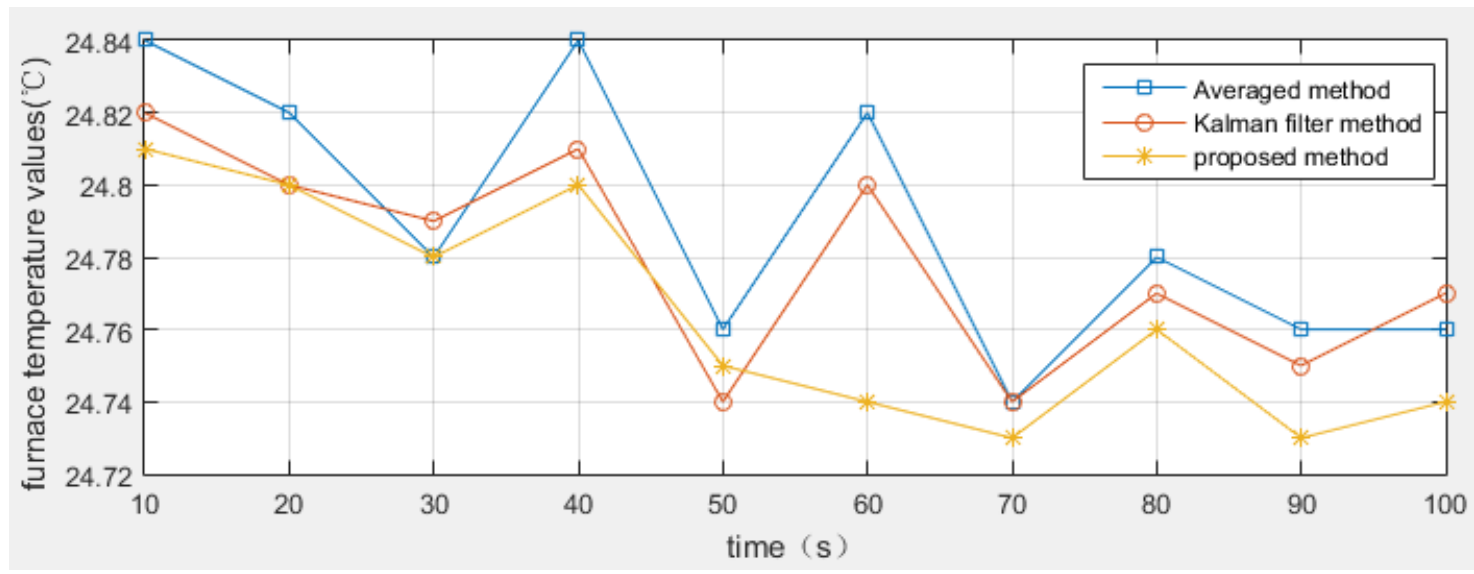

Fig.3. Comparison of three methods

\section{Conclusion}

In this paper, the average value, Kalman filter and improved Kalman filter are used to fuse the temperature values of the constant temperature furnace in the hot-press furnace. Through the simulation experiments, the improved algorithm can effectively avoid the sudden change of the data, which provides a new method for the time series data fusion in the same space, and lays a foundation for the further study of the furnace temperature data.

\section{References}

[1] Cai Xiaoqing, Lu Xiaoli, Zhang Weijuan, etc. Greenhouse monitoring system based on Kalman filter data fusion. Electronic test, 2016, 6: 59-60.

[2] Chen YK, Si XC, Li ZG. Research on Kalman-filter based multisensor data fusion. Journal of Systems Engineering and Electronics, 2007, 18(3): 497-502. [doi: 10.1016/S1004-4132 (07)60119-4]

[3] Liu Weiping, Wang Ning, Zhou Xiaolei, etc. Application of data fusion technology in the field of environmental monitoring. Computer system application, 2016, 25 (6): 88-93.

[4] Wang J, Xue HR, Jiang XH. Application of Kalman filtering algorithm in greenhouse environment monitoring. Proceedings of the 2013 2nd International Symposium on Instrumentation and Measurement, Sensor Network and Automation (IMSNA). Toronto, ON, Canada. 2013. 539544.

[5] CHEN Zhi-feng, CAI Yun-ze. Data fusion algorithm for multi-sensor dynamic system based on interacting multiple model[j]. Department of Automation, Shanghai Jiaotong University, 2015(3) : 25-29. 